\title{
Equivalence of Deterministic and Nondeterministic Epsilon Automata
}

\author{
Michał Trybulec \\ YAC Software \\ Warsaw, Poland
}

\begin{abstract}
Summary. Based on concepts introduced in [14], semiautomata and leftlanguages, automata and right-languages, and langauges accepted by automata are defined. The powerset construction is defined for transition systems, semiautomata and automata. Finally, the equivalence of deterministic and nondeterministic epsilon automata is shown.
\end{abstract}

MML identifier: FSM_3, version: $\underline{7.11 .02 \quad 4.125 .1059}$

The terminology and notation used in this paper have been introduced in the following articles: [1], [8], [2], [11], [6], [18], [7], [9], [17], [16], [15], [4], [10], [13], $[3],[12],[5]$, and [14].

\section{Preliminaries}

For simplicity, we adopt the following convention: $x, y, X$ denote sets, $E$ denotes a non empty set, $e$ denotes an element of $E, u, u_{1}, v, v_{1}, v_{2}, w$ denote elements of $E^{\omega}, F$ denotes a subset of $E^{\omega}, i, k, l$ denote natural numbers, $\mathfrak{T}$ denotes a non empty transition-system over $F$, and $S, T$ denote subsets of $\mathfrak{T}$.

One can prove the following propositions:

(1) If $i \geq k+l$, then $i \geq k$.

(2) For all finite sequences $a, b$ such that $a^{\frown} b=a$ or $b^{\frown} a=a$ holds $b=\emptyset$.

(3) For all finite sequences $p, q$ such that $k \in \operatorname{dom} p$ and $\operatorname{len} p+1=\operatorname{len} q$ holds $k+1 \in \operatorname{dom} q$.

(4) If len $u=1$, then there exists $e$ such that $\langle e\rangle=u$ and $e=u(0)$. 
(5) If $k \neq 0$ and len $u \leq k+1$, then there exist $v_{1}, v_{2}$ such that len $v_{1} \leq k$ and len $v_{2} \leq k$ and $u=v_{1} \frown v_{2}$.

(6) For all finite 0 -sequences $p, q$ such that $\langle x\rangle^{\frown} p=\langle y\rangle^{\frown} q$ holds $x=y$ and $p=q$.

(7) If len $u>0$, then there exist $e, u_{1}$ such that $u=\langle e\rangle^{\frown} u_{1}$.

Let us consider $E$. One can verify that Lex $E$ is non empty.

Next we state three propositions:

(8) \langle\rangle$_{E} \notin \operatorname{Lex} E$.

(9) $u \in$ Lex $E$ iff len $u=1$.

(10) If $u \neq v$ and $u, v \in \operatorname{Lex} E$, then it is not true that there exists $w$ such that $u^{\frown} w=v$ or $w^{\frown} u=v$.

\section{Transition Systems over Lex $E$}

The following propositions are true:

(11) For every transition-system $\mathfrak{T}$ over Lex $E$ holds \langle\rangle$_{E} \notin$ rng dom (the transition of $\mathfrak{T})$.

(12) For every transition-system $\mathfrak{T}$ over Lex $E$ such that the transition of $\mathfrak{T}$ is a function holds $\mathfrak{T}$ is deterministic.

\section{Powerset Construction for Transition Systems}

Let us consider $E, F, \mathfrak{T}$. The functor bool $\mathfrak{T}$ yielding a strict transitionsystem over Lex $E$ is defined by the conditions (Def. 1).

(Def. 1)(i) The carrier of bool $\mathfrak{T}=2^{\text {the carrier of } \mathfrak{T}}$, and

(ii) for all $S, w, T$ holds $\langle\langle S, w\rangle, T\rangle \in$ the transition of bool $\mathfrak{T}$ iff len $w=1$ and $T=w-\operatorname{succ}_{\mathfrak{T}}(S)$.

Let us consider $E, F, \mathfrak{T}$. Note that bool $\mathfrak{T}$ is non empty and deterministic.

Let us consider $E, F$ and let $\mathfrak{T}$ be a finite non empty transition-system over $F$. One can check that bool $\mathfrak{T}$ is finite.

The following two propositions are true:

(13) If $x,\langle e\rangle \Rightarrow_{\text {bool T }}^{*} y,\langle\rangle_{E}$, then $x,\langle e\rangle \Rightarrow_{\text {bool } \mathfrak{T}} y,\langle\rangle_{E}$.

(14) If len $w=1$, then $X=w-\operatorname{succ}_{\mathfrak{T}}(S)$ iff $S, w \Rightarrow_{\text {bool } \mathfrak{T}}^{*} X$. 


\section{Semiautomata}

Let us consider $E, F$. We consider semiautomata over $F$ as extensions of transition-system over $F$ as systems

$\langle$ a carrier, a transition, an initial state $\rangle$, where the carrier is a set, the transition is a relation between the carrier $\times F$ and the carrier, and the initial state is a subset of the carrier.

Let us consider $E, F$ and let $\mathfrak{S}$ be a semiautomaton over $F$. We say that $\mathfrak{S}$ is deterministic if and only if:

(Def. 2) The transition-system of $\mathfrak{S}$ is deterministic and Card (the initial state of $\mathfrak{S})=1$.

Let us consider $E, F$. One can check that there exists a semiautomaton over $F$ which is strict, non empty, finite, and deterministic.

In the sequel $\mathfrak{S}$ is a non empty semiautomaton over $F$.

Let us consider $E, F, \mathfrak{S}$. Observe that the transition-system of $\mathfrak{S}$ is non empty.

Let us consider $E, F, \mathfrak{S}$. The functor bool $\mathfrak{S}$ yields a strict semiautomaton over Lex $E$ and is defined by the conditions (Def. 3).

(Def. 3)(i) The transition-system of bool $\mathfrak{S}=\operatorname{bool}($ the transition-system of $\mathfrak{S})$, and

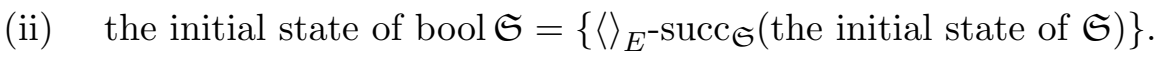

Let us consider $E, F, \mathfrak{S}$. Observe that bool $\mathfrak{S}$ is non empty and deterministic.

The following proposition is true

(15) The carrier of bool $\mathfrak{S}=2^{\text {the carrier of } \mathfrak{S}}$.

Let us consider $E, F$ and let $\mathfrak{S}$ be a finite non empty semiautomaton over $F$. Observe that bool $\mathfrak{S}$ is finite.

\section{LEFT-LANGUAGES}

Let us consider $E, F, \mathfrak{S}$ and let $Q$ be a subset of $\mathfrak{S}$. The functor left-Lang $Q$ yields a subset of $E^{\omega}$ and is defined as follows:

(Def. 4) left-Lang $Q=\left\{w: Q\right.$ meets $w$ - $\operatorname{succ}_{\mathfrak{S}}$ (the initial state of $\left.\left.\mathfrak{S}\right)\right\}$.

Next we state the proposition

(16) For every subset $Q$ of $\mathfrak{S}$ holds $w \in$ left-Lang $Q$ iff $Q$ meets $w$-succ $\mathfrak{S}$ (the initial state of $\mathfrak{S})$. 


\section{Automata}

Let us consider $E, F$. We consider automata over $F$ as extensions of semiautomaton over $F$ as systems

$\langle$ a carrier, a transition, an initial state, final states $\rangle$,

where the carrier is a set, the transition is a relation between the carrier $\times F$ and the carrier, the initial state is a subset of the carrier, and the final states constitute a subset of the carrier.

Let us consider $E, F$ and let $\mathfrak{A}$ be a automaton over $F$. We say that $\mathfrak{A}$ is deterministic if and only if:

(Def. 5) The semiautomaton of $\mathfrak{A}$ is deterministic.

Let us consider $E, F$. Observe that there exists a automaton over $F$ which is strict, non empty, finite, and deterministic.

In the sequel $\mathfrak{A}$ denotes a non empty automaton over $F$ and $p, q$ denote elements of $\mathfrak{A}$.

Let us consider $E, F, \mathfrak{A}$. One can check that the transition-system of $\mathfrak{A}$ is non empty and the semiautomaton of $\mathfrak{A}$ is non empty.

Let us consider $E, F, \mathfrak{A}$. The functor bool $\mathfrak{A}$ yields a strict automaton over Lex $E$ and is defined by the conditions (Def. 6).

(Def. 6)(i) The semiautomaton of bool $\mathfrak{A}=$ bool (the semiautomaton of $\mathfrak{A}$ ), and

(ii) the final states of bool $\mathfrak{A}=\{Q ; Q$ ranges over elements of bool $\mathfrak{A}: Q$ meets the final states of $\mathfrak{A}\}$.

Let us consider $E, F, \mathfrak{A}$. One can check that bool $\mathfrak{A}$ is non empty and deterministic.

The following proposition is true

(17) The carrier of bool $\mathfrak{A}=2^{\text {the carrier of } \mathfrak{A}}$.

Let us consider $E, F$ and let $\mathfrak{A}$ be a finite non empty automaton over $F$. Note that bool $\mathfrak{A}$ is finite.

\section{Right-LANGUAGES}

Let us consider $E, F, \mathfrak{A}$ and let $Q$ be a subset of $\mathfrak{A}$. The functor right-Lang $Q$ yields a subset of $E^{\omega}$ and is defined as follows:

(Def. 7) right-Lang $Q=\left\{w: w\right.$ - $\operatorname{succ}_{\mathfrak{A}}(Q)$ meets the final states of $\left.\mathfrak{A}\right\}$.

The following proposition is true

(18) For every subset $Q$ of $\mathfrak{A}$ holds $w \in \operatorname{right-Lang} Q$ iff $w$ - $\operatorname{succ}_{\mathfrak{A}}(Q)$ meets the final states of $\mathfrak{A}$. 


\section{Languages Accepted by Automata}

Let us consider $E, F, \mathfrak{A}$. The language generated by $\mathfrak{A}$ yielding a subset of $E^{\omega}$ is defined by the condition (Def. 8).

(Def. 8) The language generated by $\mathfrak{A}=\left\{u: \bigvee_{p, q}(p \in\right.$ the initial state of $\mathfrak{A} \wedge q \in$ the final states of $\left.\left.\mathfrak{A} \wedge p, u \Rightarrow_{\mathfrak{A}}^{*} q\right)\right\}$.

The following propositions are true:

(19) $w \in$ the language generated by $\mathfrak{A}$ if and only if there exist $p, q$ such that $p \in$ the initial state of $\mathfrak{A}$ and $q \in$ the final states of $\mathfrak{A}$ and $p, w \Rightarrow_{\mathfrak{A}}^{*} q$.

(20) $\quad w \in$ the language generated by $\mathfrak{A}$ if and only if $w$-succ $\mathfrak{A}_{\text {(the initial state }}$ of $\mathfrak{A}$ ) meets the final states of $\mathfrak{A}$.

(21) The language generated by $\mathfrak{A}=$ left-Lang (the final states of $\mathfrak{A}$ ).

(22) The language generated by $\mathfrak{A}=$ right-Lang (the initial state of $\mathfrak{A}$ ).

9. Equivalence of Deterministic and Nondeterministic Epsilon Automata

In the sequel $\mathfrak{T}$ denotes a non empty transition-system over Lex $E \cup\left\{\langle\rangle_{E}\right\}$. One can prove the following three propositions:

(23) For every reduction sequence $R$ w.r.t. $\Rightarrow \mathfrak{T}$ such that $R(1)_{2}=\langle e\rangle^{\frown} u$ and $R(\text { len } R)_{2}=\langle\rangle_{E}$ holds $R(2)_{2}=\langle e\rangle \frown u$ or $R(2)_{2}=u$.

(24) For every reduction sequence $R$ w.r.t. $\Rightarrow \mathfrak{T}$ such that $R(1)_{2}=u$ and $R(\text { len } R)_{\mathbf{2}}=\langle\rangle_{E}$ holds len $R>$ len $u$.

(25) For every reduction sequence $R$ w.r.t. $\Rightarrow_{\mathfrak{T}}$ such that $R(1)_{2}=u^{\frown} v$ and $R(\operatorname{len} R)_{\mathbf{2}}=\langle\rangle_{E}$ there exists $l$ such that $l \in \operatorname{dom} R$ and $R(l)_{\mathbf{2}}=v$.

Let us consider $E, u, v$. The functor $\operatorname{chop}(u, v)$ yielding an element of $E^{\omega}$ is defined by:

(Def. 9)(i) For every $w$ such that $w^{\frown} v=u$ holds $\operatorname{chop}(u, v)=w$ if there exists $w$ such that $w^{\frown} v=u$,

(ii) $\operatorname{chop}(u, v)=u$, otherwise.

The following propositions are true:

(26) Let $p$ be a reduction sequence w.r.t. $\Rightarrow \mathfrak{T}$. Suppose $p(1)=\left\langle x, u^{\frown} w\right\rangle$ and $p(\operatorname{len} p)=\left\langle y, v^{\frown} w\right\rangle$. Then there exists a reduction sequence $q$ w.r.t. $\Rightarrow_{\mathfrak{T}}$ such that $q(1)=\langle x, u\rangle$ and $q(\operatorname{len} q)=\langle y, v\rangle$.

(27) If $\Rightarrow \mathfrak{T}$ reduces $\left\langle x, u^{\frown} w\right\rangle$ to $\left\langle y, v^{\frown} w\right\rangle$, then $\Rightarrow \mathfrak{T}$ reduces $\langle x, u\rangle$ to $\langle y$, $v\rangle$.

(28) If $x, u^{\frown} w \Rightarrow_{\mathfrak{T}}^{*} y, v^{\frown} w$, then $x, u \Rightarrow_{\mathfrak{T}}^{*} y, v$.

(29) For all elements $p, q$ of $\mathfrak{T}$ such that $p, u^{\frown} v \Rightarrow_{\mathfrak{T}}^{*} q$ there exists an element $r$ of $\mathfrak{T}$ such that $p, u \Rightarrow \Rightarrow_{\mathfrak{T}}^{*} r$ and $r, v \Rightarrow \Rightarrow_{\mathfrak{T}}^{*} q$. 


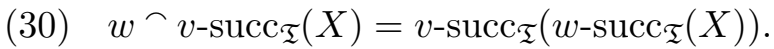

(31) bool $\mathfrak{T}$ is a non empty transition-system over Lex $E \cup\left\{\langle\rangle_{E}\right\}$.

(32) $w$ - $\operatorname{succ}_{\text {bool }}\left(\left\{v-\operatorname{succ}_{\mathfrak{T}}(X)\right\}\right)=\left\{v^{\frown} w-\operatorname{succ}_{\mathfrak{T}}(X)\right\}$.

In the sequel $\mathfrak{S}$ denotes a non empty semiautomaton over Lex $E \cup\left\{\langle\rangle_{E}\right\}$.

One can prove the following proposition

(33) $w$-succ $\operatorname{bool~}_{\mathfrak{S}}\left(\left\{\langle\rangle_{E}-\operatorname{succ}_{\mathfrak{S}}(X)\right\}\right)=\left\{w-\operatorname{succ}_{\mathfrak{S}}(X)\right\}$.

In the sequel $\mathfrak{A}$ denotes a non empty automaton over Lex $E \cup\left\{\langle\rangle_{E}\right\}$ and $P$ denotes a subset of $\mathfrak{A}$.

Next we state several propositions:

(34) If $x \in$ the final states of $\mathfrak{A}$ and $x \in P$, then $P \in$ the final states of bool $\mathfrak{A}$.

(35) If $X \in$ the final states of bool $\mathfrak{A}$, then $X$ meets the final states of $\mathfrak{A}$.

(36) The initial state of bool $\mathfrak{A}=\left\{\langle\rangle_{E}\right.$-succ $\mathfrak{A}_{\mathfrak{A}}($ the initial state of $\left.\mathfrak{A})\right\}$.

(37) $w$-succ bool $\mathfrak{A}\left(\left\{\langle\rangle_{E}-\operatorname{succ}_{\mathfrak{A}}(X)\right\}\right)=\left\{w-\operatorname{succ}_{\mathfrak{A}}(X)\right\}$.

(38) $w$-succ bool $\mathfrak{A}_{(\text {the initial state of bool } \mathfrak{A})=\{w \text {-succ }}($ the initial state of $\mathfrak{A})\}$.

(39) The language generated by $\mathfrak{A}=$ the language generated by bool $\mathfrak{A}$.

(40) Let $\mathfrak{A}$ be a non empty automaton over Lex $E \cup\left\{\langle\rangle_{E}\right\}$. Then there exists a non empty deterministic automaton $\mathfrak{A}_{1}$ over Lex $E$ such that the language generated by $\mathfrak{A}=$ the language generated by $\mathfrak{A}_{1}$.

(41) Let $\mathfrak{F}$ be a non empty finite automaton over $\operatorname{Lex} E \cup\left\{\langle\rangle_{E}\right\}$. Then there exists a non empty deterministic finite automaton $\mathfrak{A}_{2}$ over Lex $E$ such that the language generated by $\mathfrak{F}=$ the language generated by $\mathfrak{A}_{2}$.

\section{REFERENCES}

[1] Grzegorz Bancerek. Cardinal numbers. Formalized Mathematics, 1(2):377-382, 1990.

[2] Grzegorz Bancerek. The fundamental properties of natural numbers. Formalized Mathematics, 1(1):41-46, 1990.

[3] Grzegorz Bancerek. The ordinal numbers. Formalized Mathematics, 1(1):91-96, 1990.

[4] Grzegorz Bancerek. Reduction relations. Formalized Mathematics, 5(4):469-478, 1996.

[5] Grzegorz Bancerek and Krzysztof Hryniewiecki. Segments of natural numbers and finite sequences. Formalized Mathematics, 1(1):107-114, 1990.

[6] Czesław Byliński. Functions and their basic properties. Formalized Mathematics, 1(1):5565, 1990.

[7] Czesław Byliński. Functions from a set to a set. Formalized Mathematics, 1(1):153-164, 1990.

[8] Czesław Byliński. Some basic properties of sets. Formalized Mathematics, 1(1):47-53, 1990.

[9] Agata Darmochwał. Finite sets. Formalized Mathematics, 1(1):165-167, 1990.

[10] Karol Pąk. The Catalan numbers. Part II. Formalized Mathematics, 14(4):153-159, 2006, doi:10.2478/v10037-006-0019-7.

[11] Andrzej Trybulec. Domains and their Cartesian products. Formalized Mathematics, 1(1):115-122, 1990 .

[12] Andrzej Trybulec. Tuples, projections and Cartesian products. Formalized Mathematics, 1(1):97-105, 1990.

[13] Michał Trybulec. Formal languages - concatenation and closure. Formalized Mathematics, 15(1):11-15, 2007, doi:10.2478/v10037-007-0002-y. 
[14] Michał Trybulec. Labelled state transition systems. Formalized Mathematics, 17(2):163171, 2009, doi: 10.2478/v10037-009-0019-5.

[15] Zinaida Trybulec. Properties of subsets. Formalized Mathematics, 1(1):67-71, 1990.

[16] Tetsuya Tsunetou, Grzegorz Bancerek, and Yatsuka Nakamura. Zero-based finite sequences. Formalized Mathematics, 9(4):825-829, 2001.

[17] Edmund Woronowicz. Relations and their basic properties. Formalized Mathematics, 1(1):73-83, 1990.

[18] Edmund Woronowicz. Relations defined on sets. Formalized Mathematics, 1(1):181-186, 1990.

Received May 25, 2009 